\title{
Isolation of L-form Bacteria from Urine using Filtration Method
}

\author{
Frances Davison ${ }^{1}$, Jonathan Chapman ${ }^{1}$, Katarzyna Mickiewicz ${ }^{1}$ \\ ${ }^{1}$ Centre for Bacterial Cell Biology, Biosciences Institute, Medical School, Newcastle University
}

\section{Corresponding Author}

Katarzyna Mickiewicz

Katarzyna.Mickiewicz@newcastle.ac.uk

\section{Citation}

Davison, F., Chapman, J.,

Mickiewicz, K. Isolation of L-form

Bacteria from Urine using Filtration

Method. J. Vis. Exp. (160), e61380,

doi:10.3791/61380 (2020).

\section{Date Published}

June 13,2020

\section{DOI}

$10.3791 / 61380$

URL

jove.com/video/61380

\section{Abstract}

Transition of bacteria to the L-form state is thought to play a possible role in immune evasion and bacterial persistence during treatment with cell wall-targeting antibiotics. However, isolation and handling of L-form bacteria is challenging, mainly due to their high sensitivity to changes in osmolarity. Here, we describe detailed protocols for the preparation of $\mathrm{L}$-form medium, isolation of $\mathrm{L}$-forms from urine using a filtration method, detection of L-forms in urine samples by phase contrast microscopy and induction of L-forms in vitro. The exact requirements for survival and growth of L-forms may vary from strain to strain. Therefore, the methods presented here are intended to act as basic guidelines for establishing L-form protocols within individual laboratories, rather than as precise instructions. The filtration method can lead to a reduction in the number of L-forms in a sample and should not be used for quantification. However, it is the only method used so far for effective separation of cell wall-deficient variants from their walled counterparts and for identification of bacterial strains, which are capable of Lform switching in patients with urinary tract infections. The filtration method has the potential to be adapted for the isolation of L-forms from patients with other categories of bacterial infections and from environmental samples.

\section{Introduction}

Virtually all bacteria are surrounded by a structure called the cell wall. The wall is important for protection against environmental stresses, helps with regular division and gives bacteria their shape ${ }^{1}$. However, the wall is also a target for parts of the immune system and some of the best and most used antibiotics, including penicillin ${ }^{2,3}$. Despite its importance, both Gram-positive and Gram-negative bacteria can occasionally survive without the wall ${ }^{4,5,6,7,8}$. If the surrounding conditions provide enough osmoprotection to prevent them from bursting and cell wall-targeting agents are also present, bacteria can transition into a wall-less state, referred to as an L-form ${ }^{4,5,6,7,8}$.

Numerous reports indicate that switching to an L-form state and back to a walled state may be important in vivo as a mechanism for bacteria to survive both the attack from the host immune system and treatment with cell wall- 
targeting antibiotics $9,10,11,12,13,14,15$. Such a transition potentially provides a powerful strategy for the recurrence of bacterial infection $9,10,11,12,13,14,15$. Understanding the basic biology of L-form bacteria and their interactions with the host are critical to decipher their role in pathogenesis. However, handling L-form bacteria is challenging.

Firstly, due to the lack of the cell wall, L-form bacteria are prone to bursting in response to changes in osmolarity. Additionally, L-forms divide in a highly irregular manner, have unpredictable patterns of growth (usually much slower than their walled counterparts) and, depending on strain, may propagate better on semi-liquid, rather than solid or liquid media. All the above considerations make quantification and comparisons of growth rates difficult. Different bacterial species (or even strains) have diverse metabolic requirements for L-form switching and growth. For example, L-forms of certain Gram-positive bacteria, which rely on aerobic respiration, are more sensitive to reactive oxygen species than their walled counterparts ${ }^{16}$.

Induction of L-forms under laboratory conditions and in the host is usually driven by cell wall-targeting agents, such as antibiotics and lysozyme ${ }^{9}$. Such a treatment might result in only a partial cell wall loss and therefore some walled (or partially walled) bacteria could be present in the samples, making it hard to distinguish whether any observed experimental outcomes are due to the presence of L-forms or walled forms of bacteria. The frequency of Lforms induced in vivo tend to be low, meaning they can be difficult to find and isolate. Finally, owing to their polymorphic morphology, L-forms can be easily confused in situ with structures of eukaryotic origin, such as apoptotic bodies or various granules.
Since their discovery in $1935^{17}$, several methods have been developed to handle L-forms in the laboratory. Most of these rely on the addition of an osmoprotective agent to the growth medium; usually a sugar or a salt $^{9}, 10,11,12,13,14,15,16,17,18$. As mentioned above, L-forms often occur side by side with walled bacteria in patient samples and separating the two populations can be difficult. However, it has been demonstrated that, unlike walled bacteria, L-forms can pass through a $0.45 \mu \mathrm{m}$ filter due their flexibility and variable sizes. A method using such a filter has been employed to isolate L-forms from urine $10,19,20,21$.

Here, we present a protocol for the isolation of L-form bacteria from urine, using a filtration method (Figure 1). Complementary protocols for the preparation of L-form medium, microscopic observation of L-forms and induction of L-forms in vitro are also described.

\section{Protocol}

\section{Preparation of L-form medium (LM)}

1. Weigh out sucrose (amount adjusted to achieve a final concentration of $0.58 \mathrm{M}$ ), $\mathrm{MgSO}_{4}$ (final concentration $8 \mathrm{mM}$ ) and Brain Heart Infusion (BHI) (amount recommended by the supplier) in a glass bottle twice the size of the desired volume of medium. To prepare $0.5 \mathrm{~L}$ of the medium, weigh out $100 \mathrm{~g}$ of sucrose, $1 \mathrm{~g}$ of $\mathrm{MgSO}_{4}$ and $18.5 \mathrm{~g}$ of $\mathrm{BHI}$ and place in a $1 \mathrm{~L}$ glass bottle. This will allow for easier resuspension of the ingredients prior to autoclaving.

2. If solid or semi-solid media are required, add a desired amount of agar. For solid media, add $5 \mathrm{~g}$ of agar to achieve a final concentration of $1 \%$ in $0.5 \mathrm{~L}$ of medium. 
For semi-solid media, add $1 \mathrm{~g}$ of agar to achieve a final concentration of $0.2 \%$ in $0.5 \mathrm{~L}$ of medium.

3. Top off with deionized (DI) water to the final desired volume.

4. Close the bottle and mix well by shaking. There is no need to resuspend the ingredients completely, just make sure they are not clumped together at the bottom of the bottle. Loosen the cap and autoclave on a sensitive media cycle $\left(115^{\circ} \mathrm{C}\right.$ for $\left.15 \mathrm{~min}\right)$. Mixing the ingredients prior to autoclaving and using a sensitive media cycle is recommended to prevent clumping of the ingredients and sucrose caramelization.

5. Visually examine the medium following autoclaving. No clumps should be present and the medium should be amber in colour (Figure 2A).

6. Following autoclaving, let the medium cool down to a temperature which allows the bottle to be comfortably held by hand.

7. If the medium is required for the induction of L-forms, add an antibiotic and/or a lytic agent at this point (for example, phosphomycin at a final concentration of 400 $\mu \mathrm{g} / \mathrm{mL}$, penicillin $\mathrm{G}$ at $200 \mu \mathrm{g} / \mathrm{mL}$, D-cycloserine at 400 $\mu \mathrm{g} / \mathrm{mL}$, ampicillin at $100 \mu \mathrm{g} / \mathrm{mL}$, moenomycin at $50 \mu \mathrm{g} /$ $\mathrm{mL}$, lysozyme at $100 \mu \mathrm{g} / \mathrm{mL}$ or lysostaphin at $5 \mu \mathrm{g} / \mathrm{mL}$ ). Empirically establish the type of L-form inducing agent (or agents if more than one is required) and its concentration for each of the bacterial species tested.

8. To prepare individual aliquots, wear gloves and work in a microbiological safety cabinet or use a Bunsen burner. The media might require incubation for prolonged periods of time and sterility is of paramount importance.

1. To prepare aliquots of solid medium, transfer $25 \mathrm{~mL}$ to individual 92/16 $\mathrm{mm}$ Petri dishes and let them set.
Using a pipettor rather than pouring plates directly is recommended to reduce the risk of contamination. Do not overdry the plates.

2. To prepare semi-liquid aliquots, transfer $5 \mathrm{~mL}$ of medium into multiple $30 \mathrm{~mL}$ polystyrene universal containers using a pipettor and let them set.

9. Use the media immediately or store at $4{ }^{\circ} \mathrm{C}$ for up to a week before use. Check manufacturer recommendations before storing medium containing antibiotics as the concentration might decrease, due to the compound degrading over time.

\section{Isolation of L-forms from urine}

NOTE: Wear gloves and a lab coat during the laboratory procedures. Work in a microbiological safety cabinet or use a Bunsen burner. Wear safety googles during filtration.

1. At least $1 \mathrm{~h}$ before the predicted arrival of the urine samples, wipe the working area with $70 \%$ ethanol and set out the required number of semi-liquid LM medium aliquots.

NOTE: A suspension of E. coli ST144 ${ }^{10}$ L-forms induced in the laboratory in liquid LM medium, rather than human urine samples, will be used to demonstrate the protocol.

2. Wipe the working area again with $70 \%$ ethanol before processing the samples. To one side of the bench, lay out the desired number of $0.45 \mu \mathrm{L}$ cut off filters, $20 \mathrm{~mL}$ syringes and several empty sterile $30 \mathrm{~mL}$ polystyrene universal containers equal to the number of samples. Add several spare filters on top of the number of the expected urine samples, just in case more than one filter will be needed to process some of the samples. Place the LM medium aliquots and empty sterile $30 \mathrm{~mL}$ polystyrene universal containers in a stable rack in the middle of the 
bench. If microscopic examination is to be carried out in parallel, also prepare glass microscope slides, $22 \times 22$ $\mathrm{mm}$ coverslips, $2 \mu \mathrm{L}$ and $1 \mathrm{~mL}$ pipettes, sterile $10 \mu \mathrm{L}$ and $1 \mathrm{~mL}$ tips, sterile $1.5 \mathrm{~mL}$ tubes and make sure a bench microcentrifuge is available.

3. Transport urine samples from the donor to the laboratory as soon as possible following donation, to prevent potential deterioration of L-form bacteria in the sample.

4. Put on gloves and goggles, remove the urine samples from the safety bag, spray them with ethanol, wipe and place in the rack, near the LM medium aliquots and sterile spare $30 \mathrm{~mL}$ polystyrene containers. From this point, process one sample at a time.

5. Loosen the caps on a tube containing LM medium, spare polystyrene universal container and tube containing urine.

6. Remove the syringe from the package, remove the plunger and place to your right.

7. Pull the safety paper from the back of the filter package and attach the syringe firmly to the filter (keep the filter face down in the individual manufacturer plastic holder to make sure it remains sterile).

8. Working quickly but carefully, remove and discard the cap from the tube of LM medium and place the filter with the syringe on top of the tube.

9. Remove the cap from the urine sample and gently pour $\sim 10 \mathrm{~mL}$ into the syringe (only $2 \mathrm{~mL}$ will be filtered but excess volume prevents splashing), making sure over 1 $\mathrm{mL}$ is left for a microscopic examination. Only $0.5 \mathrm{~mL}$ will be used, but it is impractical to precisely measure such a volume while preforming filtration, therefore an approximate excess volume is retained.
10. Very carefully place the plunger into the back of the syringe. A small amount of resistance can be felt before the plunger is placed in a fully operational position and it is easy to spill the sample past this point. Practice several times using water before processing urine samples.

11. Gently press the plunger and pass $2 \mathrm{~mL}$ of urine through the filter until an increasing resistance is felt. Be careful not to apply too much pressure to prevent the sample from spilling over and the filter from breaking. If the sample is particularly dense and generates too much resistance, pass the sample through multiple filters, rather than forcing it through one.

12. Gently tap the filter against the tube containing LM media to dislodge any remaining filtered sample, lift the filter and the syringe and quickly place the cap from the spare $30 \mathrm{~mL}$ polystyrene universal container on the tube containing LM medium and filtered urine, making sure not to touch the inside of the tube with the edge of the cap. Safely dispose of the filter, syringe and spare polystyrene container.

13. Incubate the samples in a stationary position at $30{ }^{\circ} \mathrm{C}$ for up to 1 month. This will allow L-forms that passed through the filter to regenerate their walls and start growing as walled bacteria.

14. Observe the samples every day for evidence of growth, holding the tubes containing filtered samples against a light source. For positive samples, the growth usually appears within 3 to 7 days.

15. Once the growth has been detected, transfer the sample to a microbiological safety cabinet or within proximity of a Bunsen burner. Open the sample and carefully dip in a $5 \mu \mathrm{L}$ plastic loop, aiming for the area of growth, and then 
streak the samples on standard solid BHI medium without osmoprotection, aiming to obtain single colonies ${ }^{22}$.

16. At the same time, examine a small amount of the sample, together with a fraction of semi-liquid medium $(\sim 5 \mu \mathrm{L})$, microscopically as described in step 3 . Transferring the sample onto a microscopic slide together with the medium will ensure that any L-forms which may still be present in the sample will not burst and can be detected. However, the majority of the bacteria at this point are expected to have reverted to walled forms and appear as regular rods or cocci.

17. Incubate the samples streaked on the $\mathrm{BHI}$ plates in a stationary position at $37^{\circ} \mathrm{C}$ overnight.

18. Examine for evidence of growth, pick a single colony from each plate using a $5 \mu \mathrm{L}$ loop and inoculate into $5 \mathrm{~mL}$ of liquid $\mathrm{BHI}$. Incubate at $37^{\circ} \mathrm{C}$ overnight, with shaking.

19. Use the overnight cultures to prepare glycerol stocks or to isolate DNA for species identification using a preferred method (e.g., Qiagen DNeasy Blood \& Tissue Kit).

\section{Examination of urine samples for the presence of L-forms by phase contrast microscopy}

1. Place the remaining $0.5 \mathrm{~mL}$ of urine sample in a microcentrifuge tube and spin at 8,000 $\times g$ for $1 \mathrm{~min}$ in a bench microcentrifuge.

2. Gently remove the supernatant, leaving behind approximately $20 \mu \mathrm{L}$ and resuspend the pellet by pipetting gently 3 times up and down.

3. Place $1 \mu \mathrm{L}$ onto a glass microscope slide and cover with a $22 \times 22 \mathrm{~mm}$ coverslip (to examine a $5 \mu \mathrm{L}$ sample (see step 2.16) use a $22 \times 50 \mathrm{~mm}$ coverslip to make sure the coverslip will adhere well to the slide). Press down on the coverslip gently using a sterile cotton wool pad to create a seal and to check that the cover slip is not able to move. Discard the cotton wool pad.

4. Examine the slide using a microscope equipped with a $100 x$ phase contrast objective for the presence of $L$-formlike structures (Figure 3).

NOTE: To confirm bacterial origin of L-form-like structures in patient samples, it is recommended to examine the samples by fluorescent in situ hybridisation (FISH) using oligonucleotide probes specific for bacterial sequences $^{10,23}$.

\section{Induction of L-forms in vitro}

1. Streak walled bacteria of choice to single colonies ${ }^{22}$ on a plate containing non-osmoprotective medium (for example $\mathrm{BHI}$ ) and incubate stationary at $37^{\circ} \mathrm{C}$ overnight.

2. The next day, prepare osmoprotective LM medium, containing L-form inducing agent/s of choice as described in step 1. Prepare the desired number of plates with solid medium.

3. Using a sterile $5 \mu \mathrm{L}$ plastic loop or a toothpick, pick a generous amount ( 4-5 colonies worth) from the nonosmoprotective $\mathrm{BHI}$ plate incubated the night before.

4. Streak bacteria on the LM plate using the flat surface of the loop (rather than the edge). Streak the bacteria with one continuous motion on the edge of the plate, then turn the plate $90^{\circ}$. Touch the loop to the edge of the first streak and with a continuous motion, spread the streak over a quadrant of the plate, gradually diluting the bacteria with multiple overlapping streaks (the whole plate can be used if desired. If using only a quadrant of the plate, other quadrants can be used for multiple inductions).

NOTE: Figure 4A shows schematically the direction of streaking. The aim here is to obtain an area of growth 
containing as many pure L-forms as possible, rather than single colonies. For that reason, there is no need to change the loops between streaks. Single colonies of Lforms can be very difficult to obtain and using too small a number of bacteria could result in no L-form growth at all. On the other hand, if too many walled bacteria have been used for the induction, not all bacteria will undergo the switch and some may still retain the wall. The best Lform growth area can usually be detected in the middle of the streak.

5. Place the plate in a plastic bag or other container to prevent it from drying and incubate at $30^{\circ} \mathrm{C}$ (L-forms of some bacterial species might grow better in anaerobic conditions, so an anaerobic chamber can be tested for more efficient L-form growth). Examine the plate for evidence of growth every day. This usually appears within 3 to 7 days. The amount of growth is strain specific. An example of $L$-form growth is shown in Figure 4B.

6. Confirm the presence of L-forms using a microscope. Place a $2 \mu \mathrm{L}$ droplet of $\mathrm{LM}$ medium on a microscopic slide and, using a pipette tip, pick a small amount of cells from the middle of the streak and resuspend in the droplet.

1. Cover with a $22 \times 22 \mathrm{~mm}$ coverslip and examine for the presence of L-forms using a microscope with a 100x phase contrast objective. A representative example can be found in Figure 4C.

\section{Representative Results}

L-form media containing sucrose can undergo varying degrees of caramelization following autoclaving, which are associated with a change in color. Figure 2 shows representative outcomes of autoclaving media containing sucrose. Figure 2A shows a typical example of LM medium, which has turned amber in color following the autoclaving. Figure 2B shows a typical example of $1.16 \mathrm{M}$ sucrose solution ( $2 \mathrm{x}$ concentration required for making LM medium) following autoclaving. Occasionally, LM medium or sucrose may undergo extensive caramelization during autoclaving, and it is not recommended to use the medium if this happens. Figure 2C shows an example of over-caramelized sucrose medium.

L-form bacteria can be highly heterogeneous and Figure 3 shows examples of L-form-like structures observable in patient urine samples. To confirm the bacterial origin of the L-form-like structures found in urine samples, FISH with fluorescent probes targeting bacterial sequences is recommended ${ }^{10,23}$.

Growth levels of L-forms induced under laboratory conditions are strain specific. Figure 4B shows an example of Bacillus subtilis L-form growth and Figure 4C shows the microscopic appearance of L-forms induced in Figure 4B. 


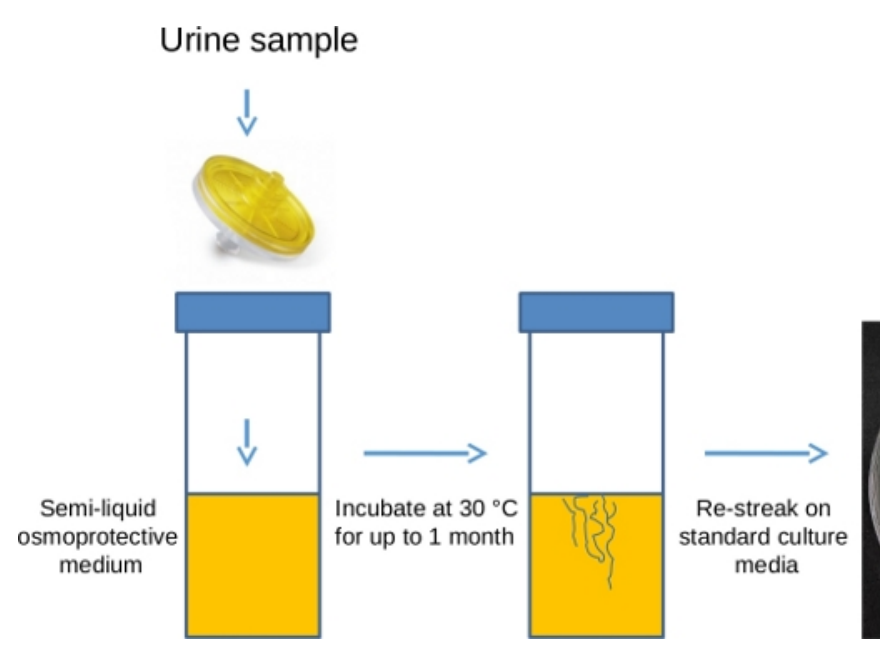

Strain identification

DNA isolation
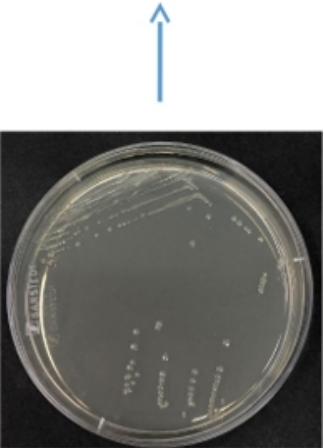

Figure 1: Isolation of L-forms using filtration method - schematic representation. Urine sample is passed through a $0.45 \mu \mathrm{m}$ filter into a polystyrene universal container, containing osmoprotective LM medium supplemented with $0.2 \%$ agar. The sample is then incubated in a stationary position at $30^{\circ} \mathrm{C}$ for up to a month and visually checked daily for evidence of growth. This incubation period allows any L-forms that are present in the sample to regenerate their cell walls. Once the growth is detected, the bacteria can be re-streaked on a plate containing regular, solid, non-osmoprotective medium (such as nutrient agar or brain-heart infusion) to isolate single colonies, which can then be subjected to DNA extraction and sequencing to identify the isolated bacterial species. Please click here to view a larger version of this figure.
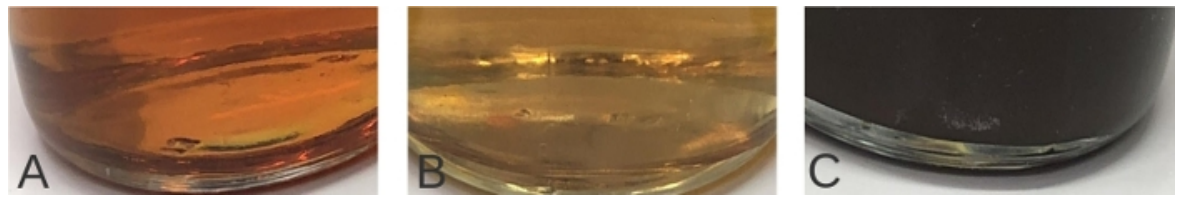

Figure 2: L-form media. (A) LM medium following autoclaving. (B) $1.16 \mathrm{M}$ sucrose (2 $\mathrm{x}$ concentration) following autoclaving.

(C) Extensively caramelized LM medium. Please click here to view a larger version of this figure. 

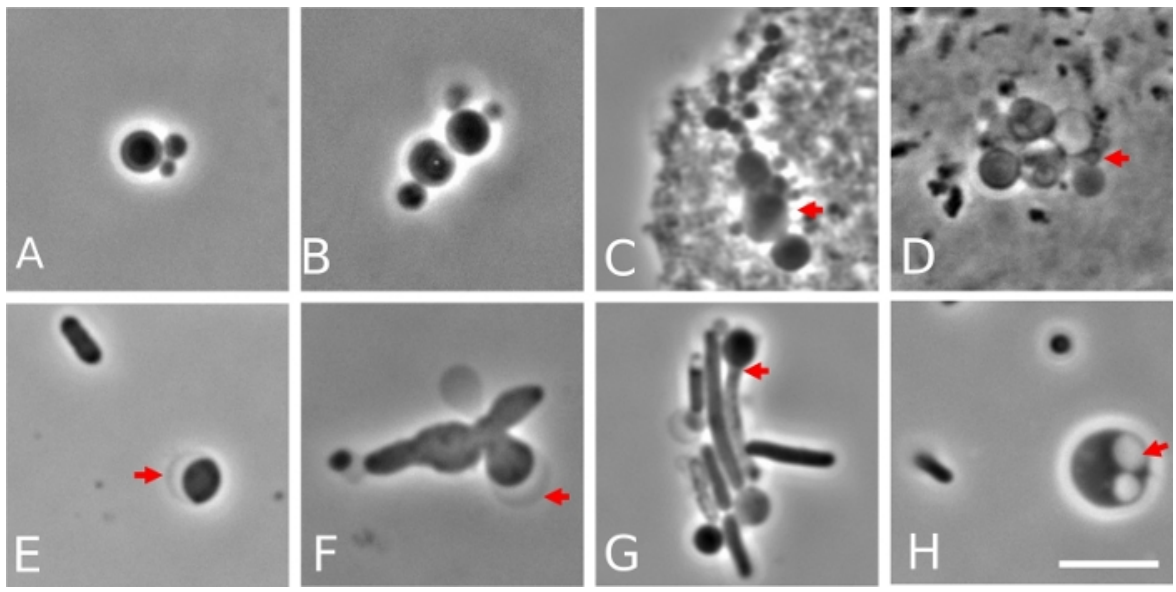

Figure 3: Examples of L-form like structures observable in urine of patients with recurrent UTI by phase contrast microscopy. (A,B) Structures typical of dividing bacterial L-forms suspended in urine. (C,D) Structures typical of dividing bacterial L-forms associated with eukaryotic cells. (E,F) Crescent shaped structure characteristic for Gram-negative L-forms (red arrow). (F,G) Structures typical for intermediate stages of transition between the walled cells and L-forms (red arrow in G). (H) Intracellular vesicles typical for large L-forms. Scale bar $=5 \mu \mathrm{m}$. This figure has been modified from ${ }^{10}$. Please click here to view a larger version of this figure.
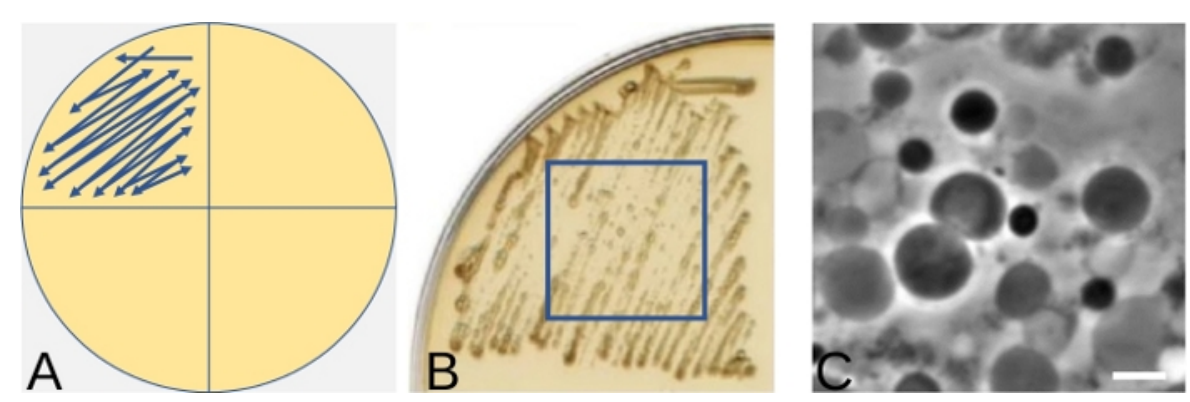

Figure 4: Streak-plate technique for induction of L-forms on solid media. (A) Schematic representation of streaking bacteria using a quarter of a plate for induction of L-forms. The arrow indicates the direction to streak the bacteria. (B) An example of Bacillus subtilis L-form growth following streaking, as shown in (A), after 3 days incubation at $30^{\circ} \mathrm{C}$. (C) L-forms induced in (B) visualized by phase contrast microscopy. Scale bar $=5 \mu \mathrm{m}$. Panels $B$ and $C$ have been modified from ${ }^{16}$ Please click here to view a larger version of this figure. 


\section{Discussion}

Protocols described in this manuscript have been used to isolate and handle L-forms of various bacterial species from human urine, including E. coli, Streptococcus, Staphylococcus, Klebsiella, Pseudomonas, Enterococcus and Enterobacter spp, all of which are typically associated with UTIs ${ }^{10,24}$. However, methods for handling L-forms, established in one laboratory may not work immediately in another and what works for one bacterial strain may not work for another. Therefore, the protocols described here are likely to require multiple attempts and additional optimization. In particular, nutrient requirements, oxygen availability, osmoprotectant concentration and fluidity of the medium may need to be tested. Conditions optimal for Lform transition and growth could be different from conditions optimal for growth of walled forms of the same species. Moreover, several technical issues could be encountered when attempting the protocols.

A common problem associated with preparation of L-form media is sucrose caramelization following autoclaving. If the medium turns dark brown in color, it should be discarded and a fresh batch should be prepared. It may be necessary to prepare the sucrose in water in one bottle and the remaining ingredients in another, both at $2 x$ concentration. $2 x$ concentrated solutions can then be combined at a $1: 1$ ratio following autoclaving, to achieve the desired final concentrations. Sucrose autoclaved separately may turn slightly yellow in color (Figure 2B), which is acceptable, but if it turns dark brown (Figure 2C), it should not be used for experiments. Adjusting the length and the temperature of the autoclaving cycle might be necessary to alleviate sucrose caramelization. Testing sterility of the media prepared using an adjusted autoclave cycle, by incubating an aliquot of each of the media for at least 3 days at $37{ }^{\circ} \mathrm{C}$, is recommended. Finally, it might be necessary to test an alternative osmoprotective agent, such as salt, if the problem with caramelization persists ${ }^{18}$.

Several issues might also be encountered during isolation of L-forms from patient samples. As mentioned in the protocol, L-forms present in the samples might potentially deteriorate; therefore, it is important to transport the samples to the laboratory as soon as possible after donation. For the same reason, storing samples at low temperatures or modifying sample composition (for example by addition of PBS) is strongly discouraged.

The filtration method itself has its limitations. Some L-forms may be ripped apart due to shear forces generated by the flow of media through the filter. In the study by Mickiewicz et al., only $41 \%$ of L-forms in control samples, which contained laboratory induced E. coli L-forms, passed through the filter $^{10}$. This demonstrates that the filtration method can lead to an underestimation of the number of positive samples and is not recommended for quantitative studies.

On very rare occasions, following filtration, a significant growth might be observable the next day in the semi-liquid media aliquots used for L-form isolation. This could be an indication that either the filter broke during the protocol, allowing the passage of walled bacteria, or the sample was contaminated. It is good practice to discard such samples or at least treat them with caution. Daily visual examination of the samples is critical to prevent false positives. Before processing urine samples by filtration, it is recommended to pass several samples of laboratory induced L-forms, walled bacteria and sterile medium through the filter of choice, to control for efficiency of L-form separation, potential passage 
of walled bacteria due to filter breakage and to make sure the sterile technique used is working well.

In rare cases, stable L-forms might be isolated by filtration, with no walled forms detectable by microscopy. To maintain viable L-form bacteria, it is recommended to transfer several "loop-fulls" of the sample to a tube containing fresh semi-liquid LM medium or to re-streak on solid osmoprotective medium every 3-7 days, depending on efficiency of the growth. If no walled forms appear after several passages, freezing samples in $40 \%$ glycerol at $-80{ }^{\circ} \mathrm{C}$ could be attempted to preserve the isolate; however, some L-forms may not tolerate such a procedure well.

Despite its limitations, the filtration method is the only one used so far for the separation of L-forms from walled forms in patient samples. It allows for the identification of bacterial strains capable of L-form switching in vivo. There is the potential to develop and adapt the filtration method for isolation of L-forms from other types of human or environmental samples (for example blood or plants).

Taking into account all of the above considerations, we recommend the protocols outlined in this manuscript as a good starting point for developing tailored L-form protocols in individual laboratories, rather than as rigid instructions. Working with L-forms requires great care, dedication and patience but with practice, it can be extremely rewarding. We hope that the guidelines outlined in this manuscript will become a benchmark for developing L-form protocols and will encourage more basic and clinical research groups to investigate these fascinating bacterial forms.

\section{Disclosures}

The authors have nothing to disclose.

\section{Acknowledgments}

This work was funded by a European Research Council (grant number 670980) to Jeff Errington (Director of The Centre for Bacterial Cell Biology, Newcastle University).

\section{References}

1. Typas, A., Banzhaf, M., Gross, C. A., Vollmer, W. From the regulation of peptidoglycan synthesis to bacterial growth and morphology. Nature Reviews Microbiology. 10, 123-136 (2011).

2. Wolf, A. J., Underhill, D. M. Peptidoglycan recognition by the innate immune system. Nature Reviews Immunology. 18, 243-254 (2018).

3. Kohanski, M. A., Dwyer, D. J., Collins, J. J. How antibiotics kill bacteria: from targets to networks. Nature Reviews Microbiology. 8 (6), 423-435 (2010).

4. Leaver, M. et al. Life without a wall or division machine in Bacillus subtilis. Nature. 457, 849-853 (2009).

5. Mercier, R., Kawai, Y., Errington, J. General principles for the formation and proliferation of a wall-free (L-form) state in bacteria. eLife. 3, 642 (10.7554/eLife.04629) (2014).

6. Cross, T. et al. Spheroplast-Mediated Carbapenem Tolerance in Gram-Negative Pathogens, Antimicrobial Agents and Chemotherapy. 63 (9) e00756-19 (2019).

7. Ramijan, K. et al. Stress-induced formation of cell wall-deficient cells in filamentous actinomycetes. Nature Communications. 9, 5164 (2018).

8. Errington, J. et al. L-form bacteria, chronic diseases and the origins of life. Philosophical Transactions of the Royal Society of London. Series B, Biological Sciences. 371, 20150494 (2016). 
9. Kawai, Y., Mickiewicz, K., Errington, J. Lysozyme counteracts $\beta$-Lactam antibiotics by promoting the emergence of L-form bacteria. Cell. 172, 1038-1049 (2018).

10. Mickiewicz, K.M. et al. Possible role of L-form switching in recurrent urinary tract infection. Nature Communications. 10, 4379 (2019).

11. Domingue, G. J. Sr., Woody, H. B. Bacterial persistence and expression of disease. Clinical Microbiology Reviews. 10, 320-344 (1997).

12. Clasener, H. Pathogenicity of the L-phase of bacteria. Annual Review of Microbiology. 26, 55-84 (1972).

13. Onwuamaegbu, M. E., Belcher, R. A., Soare, C. Cell walldeficient bacteria as a cause of infections: a review of the clinical significance. Journal of International Medical Research. 33, 1-20 (2005).

14. Allan, E. J., Hoischen, C., Gumpert, J. Bacterial L-forms. Advances in Applied Microbiology. 68, 1-39 (2009).

15. Domingue, G. J. Demystifying pleomorphic forms in persistence and expression of disease: Are they bacteria, and is peptidoglycan the solution? Discovery Medicine. 10, 234-246 (2010).

16. Kawai, Y. et al. Crucial role for central carbon metabolism in the bacterial L-form switch and killing by $\beta$-lactam antibiotics. Nature Microbiology. 4, 1716-1726 (2019).

17. Klieneberger, E. The natural occurrence of pleuropneumonia-like organisms in apparent symbiosis with Streptobacillus moniliformis and other bacteria. The Journal of Pathology and Bacteriology. 40, 93-105. (1935).
18. Osawa, M., Erickson, H. P. L form bacteria growth in low-osmolality medium. Microbiology. 165 (8), 842-851 (2019).

19. Domingue, G. J., Schlegel, J. U. The possible role of microbial L-forms in pyelonephritis. The Journal of Urology. 104, 790-798 (1970).

20. Braude, A. I., Siemienski, J., Jacobs, I. Protoplast formation in human urine. Transactions of the Association of American Physicians. 74, 234-245 (1961).

21. Kalmanson, G. M., Hubert, E. G., Guze, L. B. Production and therapy of Proteus mirabilis pyelonephritis in mice undergoing chronic diuresis. Antimicrobial Agents and Chemotherapy. 9, 458-462 (1969).

22. Sanders, E.R. Aseptic Laboratory Techniques: Plating Methods Journal of Visualized Experiments. 63, e3064 (2012).

23. Takada, T., Matsumoto, K., Nomoto, K. Development of multi-color FISH method for analysis of seven Bifidobacterium species in human feces. Journal of Microbiological Methods. 58, 413-421 (2004).

24. Drage, L. K. et al. Elevated urine IL-10 concentrations associate with Escherichia coli persistence in older patients susceptible to recurrent urinary tract infections. Immunity \& Ageing. 16, 1-11 (2019). 